\title{
Auditory Screening
}

\author{
Fanelli Karina* \\ Lic. In Speech Therapy, Specialist in Audiology, Buenos Aires, Argentina
}

*Corresponding author: Fanelli Karina, Lic. In Speech Therapy, Specialist in Audiology, Buenos Aires, Argentina.
Received Date: August 10, 2020

Published Date: August 25, 2020

\section{Short Communication}

The high prevalence of hearing loss in newborns and preschoolers around the world leads to audiology to design "auditory screening protocols" and "audiological follow-up". These programs, based on scientific evidence and clinical experience, were modified over time to optimize time and minimize errors in test results implemented for detection. Their objectives are to reduce the age of identification, diagnosis, and effective interventions to improve language and social-emotional outcomes in children who are deaf or hard of hearing.

Nowadays we use universal newborn hearing screening and suggest applying the screening protocol proposed by the Joint Committee on Infant Hearing (JCIH) published in the Year 2019 Position Statement: Principles and Guidelines for Early Hearing Detection and intervention Programs. "Optimal Early Hearing Detection and Intervention (EHDI) programs have been defined as meeting the EHDI 1-3-6 goals. Since 2019, to provide appropriate access to language stimulation and intervention services as soon as possible, EHDI programs meeting current targets might consider setting a new target of 1-2-3 months (screening completed by one month of age, audiologic diagnosis completed by two months of age, and early intervention initiated no later than three months of age)" [1].

Each screening center should identify and define the referral pathway to access to a pediatric audiologist who can complete the rescreening and diagnosis according to the EHDI 1-3-6 or 1-2-3 goals based on infrastructure (equipment, technologies, personnel, population). We must consider that "it may not be appropriate to apply this timeline to infants receiving care in the NICU. Because the majority of very preterm infants may still be in the NICU at 3 months of age, a recommendation is made that for very preterm infants with prolonged hospitalization, a diagnostic audiologic evaluation prior to discharge from the NICU be completed [1].

To detect deafness or hearing loss, we support the idea of using of automated OAE (either transient-evoked OAEs (TEOAE) or distortion-product OAEs (DPOAE)) and auditory brainstem response (ABR) [1,2,3]. Both automated OAE and ABR technologies provide non-invasive recordings of different structures of the auditory system, with high specificity and speed. The use of $\mathrm{OAE}$ as a screening tool is likely to result in a higher failing rate in the immediate post-birth period when compared to AABR [3]. Although, middle-ear measure should be included in the test battery to assess any possibility of a conductive component to the hearing loss $[1,4]$. At the time of newborn hearing screening, neonates may have retained amniotic fluid in the middle ear space, resulting in a not-pass outcome. The standard measure for detecting middle ear fluid has long been high frequency tympanometry, due to superior sensitivity and specificity in detecting middle ear fluid or effusion in infants when compared to standard $226 \mathrm{~Hz}$ tympanometry. The use of the 1000 probe tone is recommended up to age 9 months [1].

Clinical interpretation of DPOAEs in infants can be improved by using age-appropriate normative ranges and optimized cut-off values. DPOAE interpretation is more predictive at higher $\mathrm{f} 2$ test frequencies in young infants $(2-8 \mathrm{kHz})$ due to poor test performance at $1-1.5 \mathrm{kHz}$ [4]. After that, in the audiological diagnosis stage, the $\mathrm{ABR}$ and a behavioral audiogram is necessary to confirm the degree, type and configuration of hearing loss to guide the therapeutic intervention which consists in the first instance in the use of the hearing aids and language interventions. Depending on language 
progress and auditory performance outcomes after 6 month of hearing aids use, it will be determined whether the therapeutic intervention will be maintained or modified, resulting to a cochlear implant or bone conduction device.

Regarding the rest of the population, an auditory follow-up should be done to the rest of population who passed the newborn screening to detect late-onset hearing loss in time [1,2]. For these reasons, we suggest applying pre-school and school auditory screening to minimize the impact of hearing loss on language development and/or academic learning. The protocols would be in accordance with the characteristics of the institution in which this type of screening would take place. The most economic, effective, sensitive, and quick preschool/school hearing screening protocol could be constituted by distortion-product OAEs (DPOAE) and Tympanometry [2]. For the reasons explained above, we can conclude that auditory screening will consequently promote a better quality of life.

\section{Acknowledgement}

None.

\section{Conflict of Interest}

No conflict of interest.

\section{References}

1. (2019) The Journal of Early Hearing Detection and Intervention 4(2).

2. Hill J, De Wet S (2010) Objective assessment of hearing, CA: Plural Publishing, San Diego, USA.

3. Van Dyk M, Swanepoel de W, Hall JW $3^{\text {rd }}$ (2015) Outcomes with OAE and AABR screening in the first $48 \mathrm{~h}$--Implications for newborn hearing screening in developing countries. Int J Pediatr Otorhinolaryngol 79(7): 1034-1040.

4. Blankenship (2019) Ear Hear. 19. 